\title{
Biodegradable microplastics (BMPs): a new cause for concern?
}

\author{
Can Wang ${ }^{1} \cdot$ Jiefa Yu ${ }^{1} \cdot$ Ying $\mathrm{Lu}^{1} \cdot \mathrm{Di} \mathrm{Hua}^{2} \cdot$ Xiao Wang $^{2} \cdot$ Xuehua Zou $^{1}$
}

Received: 16 December 2020 / Accepted: 5 September 2021 / Published online: 16 September 2021

(C) The Author(s), under exclusive licence to Springer-Verlag GmbH Germany, part of Springer Nature 2021

\begin{abstract}
With the increasingly serious pollution of plastics, biodegradable plastics (BDPs) have attracted attention as a new material that can replace conventional plastics in certain applications. The global production of BDPs also gradually increases in recent years. However, unfortunately, with the application of BDPs, some potential problems are gradually exposed. The biodegradability of BDPs needs suitable conditions, which is difficult for the natural environment to reach the necessary conditions. If the degradation conditions are not met, BDPs and conventional plastics are basically the same in terms of the longevity. The biodegradable microplastics (BMPs) can also be formed by BDPs entering the environment. Up to now, the research on the degradation and application of BDPs is relatively common. The environmental and ecological effects of the BMPs, the adsorption and release of toxic substances, and the role of BMPs as vectors of microorganisms, epiphytes, and plants still need to be studied. This paper focuses on the formation mechanism and the environmental behavior of BMPs. The role of BMPs as multiple stronger vectors of microorganisms and pollutants compared to conventional microplastics is also discussed. Systematic research on environmental pollution and ecotoxicology of BMPs should be carried out as soon as possible.
\end{abstract}

Keywords Biodegradable plastic $\cdot$ Biodegradable microplastics $\cdot$ Microplastics $\cdot$ Stronger vector $\cdot$ New focus

\section{Introduction}

In recent decades, microplastics, a new type of pollutant, have attracted worldwide attention. Microplastic pollution has become a major global environmental problem in parallel with global climate change and ozone depletion (Shen et al. 2020). A large amount of evidence showed that microplastics might become a lasting problem (González-Pleiter et al. 2021). Microplastics have spread all over the world, in freshwater

\section{Highlight}

- BDPs as a promising solution to plastic pollution have been widely concerned.

- BMPs may persist in the environment for a long time.

- BMPs can act as multiple stronger vectors of microorganisms and pollutants.

- Systematic research on BMPs should be carried out as soon as possible.

Responsible Editor: Philippe Garrigues

Xuehua Zou

zouxuehua1988@hfut.edu.cn

1 School of Resources and Environmental Engineering, Hefei University of Technology, Hefei 230009, China

2 Automotive Engineering Corporation, Nankai District, Tianjin 300113, China
(Shen et al. 2019a; Wang et al. 2016), in the atmosphere (Gasperi et al. 2018), in soil (Machado et al. 2018), in sediment (Wang et al. 2019), in seafood (Catarino et al. 2018), in the oceans (Thompson et al. 2004), even in bottled water we drink (Oßmann et al. 2018). Due to the lack of enzymes to degrade microplastics, microplastics will accumulate in the digestive system. Microplastics can damage the digestive tract and stomach of organisms, and long-term intake may reduce the feeding rate of organisms (Gregory 2009). Because of the wide distribution of various organic and inorganic pollutants in natural water, the combination of these pollutants and microplastics may cause serious harm to the ecology and human health (Shen et al. 2021; 2019b). The persistent existence of microplastics in the environment may not only inhibit some plant growth, change-related microbial enzyme activities and increase biological mortality, but also accumulate in some organisms (Shen et al. 2019a). At present, the world has taken measures to solve the problem of plastic waste, such as banning the production and use of disposable plastic products and zero-waste systems (Hamilton et al. 2019). However, these methods are only partially effective, as the scale of plastic production is far greater than the reduction of these treatment methods (Hu et al. 2019).

Recently, more attention has been paid to biodegradable plastics (BDPs) because they can reduce the environmental 
pollution caused by nonbiodegradable plastics. Fig. 1 shows the global production of BDPs in the last few years. Generally, BDPs are derived from petrochemical and biobased sources (corn starch, lignin, cellulose, etc.) to reduce the dependence on oil resources and that both are available on the consumer market, which has little impact on the environment (Haider et al. 2019). After the end of the product life cycle, it is easy to handle, with compostability and biodegradability. Biodegradable plastics are a relatively small subset of bioplastics that can be converted into $\mathrm{H}_{2} \mathrm{O}, \mathrm{CO}_{2}$, and biomass with assistant of microorganisms over time. The above process is called biodegradation. BDPs have been promoted as alternative materials for disposable plastics in some industries and places. However, unfortunately, with the application of BDPs, some potential problems are gradually exposed. The biodegradability of BDPs is not unconditional. A major problem with these plastics is that they may be biodegradable, but this process requires appropriate conditions and microorganisms that are not always reliable under environmental conditions, such as suitable temperature, humidity, and microorganisms (Pico and Barcelo 2019). If the degradation conditions are not met, BDPs and conventional plastics are basically the same in terms of the longevity; they will also become plastic garbage and microplastics (Wei et al. 2021), which will pollute the water and soil. Under the effects of wave erosion, sunlight, and biodegradation, biodegradable plastic waste will be gradually decomposed into small pieces and left in the ocean to form biodegradable microplastics (BMPs). BMPs may have similar properties with conventional microplastics and can be used as vectors of pollutants and microorganisms in the environment (Zuo et al. 2019). We do not believe that BDPs are more environmentally friendly than conventional plastics without degradation (Napper and Thompson 2019; Narancic and O'Connor 2019).

The existing studies have shown that the adsorption of BMPs to pollutants is much higher than that of conventional microplastics (Zuo et al. 2019), and they may become stronger vectors. The research on the ecological impact of BMPs is a new research topic, and there is no in-depth study. Many conclusions are still in the speculative stage, and there is no sufficient data support. In this paper, the formation mechanism of BMPs and their long-term persistence in the environment are raised BMPs as stronger vectors of microorganisms and pollutants are also thoroughly discussed, and some perspectives are proposed.

\section{What is the formation mechanism of BMPs?}

BDPs are a new type of bioplastic, which refers to plastics produced by microorganisms based on starch and other natural substances and can be biodegraded naturally in the environment. They can be decomposed into substances (carbon dioxide, water, methane, and biomass) by microorganisms (bacteria, fungi, algae, etc.) existing in nature and can be integrated into the natural ecosystem without producing ecological toxicity and residual by-products. The ideal BP is a kind of polymer material with excellent performance, fast degradation, and eventually existing in nature as part of the carbon cycle. The standard test methods for the maximum duration of complete biodegradation in various environments are also different. According to the degree and nature of biodegradation, BDPs can be divided into two types, partially BDPs and fully BDPs. Biodegradation is the process of degrading BDPs into oligomers, monomers, or carbon dioxide and water by using organisms (bacteria, fungi, etc.) and finally entering the ecosystem. As a matter of fact, BDPs can be biodegraded, but the degradation process requires specific conditions and is not always reliable under natural conditions. When BDPs enter the water environment, the deterioration rate is low. One research done by Nazareth et al. (2019) explored the aging process and mechanism of BDPs in the marine environment. The results demonstrated that no obvious sign of degradation was detected, although the product was claimed to be $100 \%$ biodegradable after submerged in seawater for 180 days. Another similar report performed by Napper and Thompson (2019) also showed that there was no significant difference in oxo-biodegradable plastics before and after immersion in seawater for 3 years. When BDPs cannot be completely degraded in the environment, they can no longer be regarded as BDPs.
Fig. 1 Global production and forecast of biodegradable plastics. (A) Shows the annual production of biodegradable plastics worldwide, and (B) shows the global productivity structure of biodegradable plastics in 2018 , respectively (annual production, proportion) Zhongyan Puhua Company (2014).
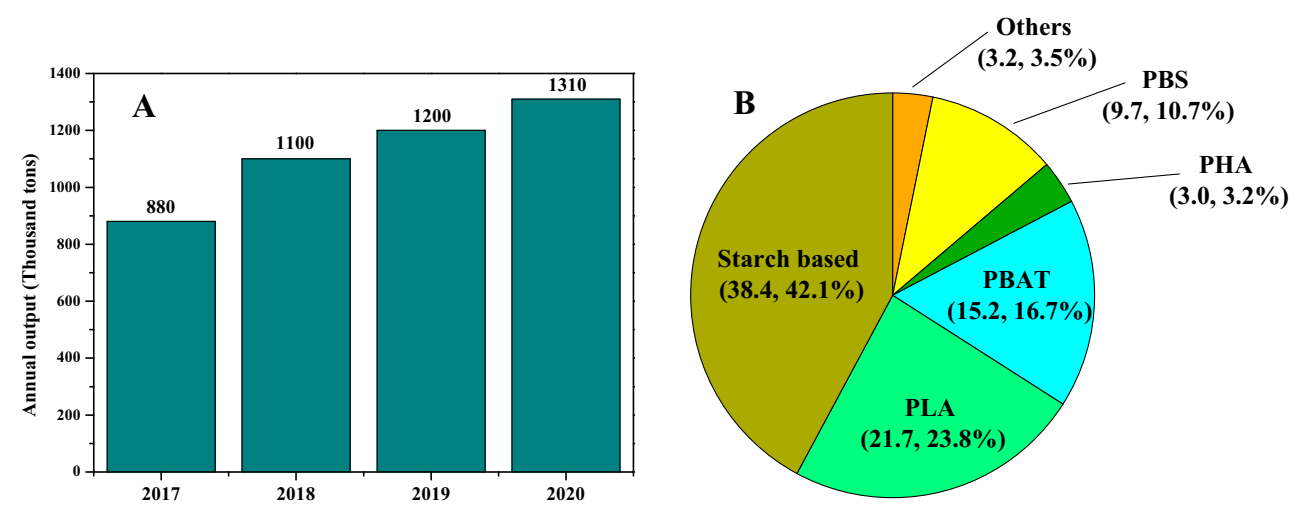
The BMPs in the water environment mainly come from the fragmentation and decomposition of large plastic debris. BDPs can migrate with wind or rain, enter surface water from the land, and finally enter the freshwater and marine ecosystems (Law and Thompson 2014; Xia et al. (2020). After the BDPs enter the water, they will be slowly broken and decomposed under the action of ultraviolet radiation, water disturbance, wave impact, physical wear, biological habitat, and freeze-thaw cycle and gradually form BMPs (Fig. 2). Microplastics can continue to decompose to form nanoplastics (Zhang et al. 2020). The process of forming microplastics from large plastic fragments is fast, but the process from the degradation of microplastics to smaller particles and mineralization is very slow. Ultraviolet radiation is the main driving force for the formation of microplastics. Generally, photooxidation degradation is relatively efficient on beach surface, but low in deep-sea or river bottom sediments, so the abundance of microplastics in different environmental media is quite different. Recently, a study done by Wei et al. (2021) showed that the microplastic risk from biodegradable polymers is high and needs to be further evaluated with regard to longer timeframes, the biological fate of intermediate products, and final products in freshwater, estuarine and seawater natural habitats. Especially, considering that these B may have good biodegradability in warmer $20-25^{\circ} \mathrm{C}$ water but will most likely be highly persistent in the cold deep seas.

BMPs possess the capabilities of conventional microplastics until they are completely mineralized. Due to its small size in water, BMPs can be easily ingested by zooplankton, filter feeders, and benthic organisms, leading to blockage of the digestive system of marine animals (Cole et al. 2013). In addition to physically blocking the digestive system, the persistent toxic pollutants contained in BMPs have toxicity and potential bioaccumulation, which can be absorbed and accumulated by the consumers and produce toxic biochemical effects. Obviously, more research is needed to understand the potential scale and scope of the problem.
Fig. 2 The possible formation mechanism and behavior of BMPs in the marine environment

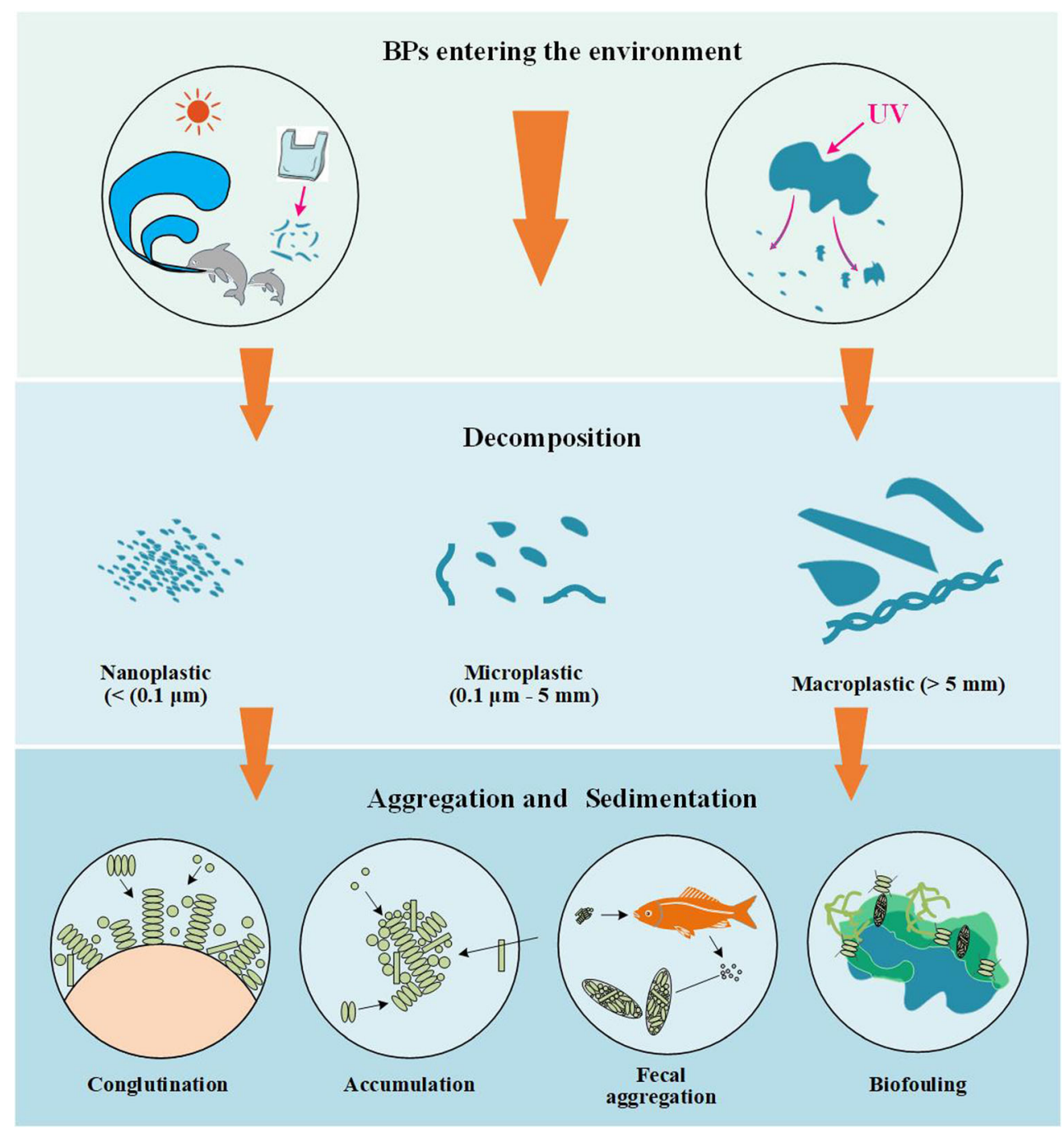




\section{Can BMPs persist in the environment?}

Degradation requires specific conditions, such as $\mathrm{pH}$, temperature, and sunlight (Napper and Thompson 2019; Pico and Barcelo 2019). As a kind of suspended particles, microplastics can adsorb organic matters and inorganic nutrients in the water, so as to attract bacteria and viruses, and other microorganisms to attach to them. This contamination process can affect BMPs in a number of ways. Biofilm can block the photodegradation of BMPs by ultraviolet radiation. Evidence showed that biofilm can change the buoyancy and viscosity of the floating microplastics, weaken the hydrophobicity (Lott et al. 2020) and cause the microplastics to settle in the deep sea or even be buried in the sediments (Lobelle and Cunliffe 2011). The sinking of BMPs further reduces the influence of ultraviolet radiation. When BMPs reach the deepwater area, the degradation rate will become slower due to the low temperature, low light, and low microbial activity. There are a lot of microorganisms in biofilm, which may have the ability to degrade BMPs. Many factors affect the biodegradation process: microbial species, initial biomass, and plastic surface area (and surface area: volume ration). Increased biomass and mineralization of organic compounds are important steps in microbial degradation of BMPs. The main chain of BMPs breaks down, and its molecular weight gradually decreases. Finally, it becomes a monomer through enzymatic hydrolysis, hydrolysis, or microbial synergy. Energy source and supply are the key to the process of degradation, whether these microorganisms only degrade BMPs or use the chemicals in BMPs (carbon source) to participate in metabolism remains unknown. However, unfortunately, the research on whether BMPs can persist in the environment is still ongoing. Many conclusions are still in the speculative stage, and there is no sufficient data to support them. Therefore, we should not only pay attention to the degradation of BDPs on the macro level but also focus on the potential impact of microplastics and nanoplastics on the environment.

\section{Can BMPs act as stronger vectors for chemical pollutants and microorganisms?}

It is assumed that the existence of BMPs is not "persistent," but it still has the characteristics of microplastics before being completely assimilated into water and carbon dioxide. Then, it will become a new kind of biological and chemical vector. Microplastics have a large specific surface area and good hydrophobicity, which have been proved to be able to enrich organic pollutants in water (Gouin et al. 2011; Koelmans et al. 2016; Ma et al. 2016; Rochman et al. 2014). At the same time, plasticizers, dyes, and other chemical components are often added in the process of plastic production in order to meet the needs of different applications. These additives may be released in the process of plastic decomposition and migrate with the flow of microplastics. Additionally, the surface of microplastics is easily covered by biofilm, which is a good place for insects to lay eggs and colonize bacteria and algae. The multi-carrier of microplastics has become an important topic (Shen et al. 2019b). Due to the similar properties, BMPs may become a stronger carrier of pollutants and microorganisms (Fig. 3).

Firstly, BMPs can adsorb more chemical pollutants in water than that of conventional microplastics. Zuo et al. (2019) explored the adsorption of organic pollutant (phenanthrene) onto BMPs (poly(butylene adipate co-terephtalate, PBAT) and conventional microplastics (PE and PS). The experimental results showed that the adsorption capacity of the degradable microplastics to phenanthrene was far greater than that of the conventional microplastics. The adsorption capacity of the degradable microplastics was 3 times of that of PE and 27 times of that of PS. The findings demonstrated that BMPs could act as stronger vectors than conventional ones in the environment. Although microplastics have good adsorption properties for pollutants, their migration ability is not high (Tong et al. 2021). By establishing a plastic migration model, the researchers predicted that the migration of microplastics was 4-6 orders of magnitude lower than that of other substances migrating with ocean current (Zarfl and Matthies 2010). Therefore, the ecological risk of microplastics and coexisting pollutants to in situ aquatic organisms may be higher. When BMP particles adsorbed pollutants are preyed on by aquatic organisms, there is a risk of joint exposure (Fig. 3). In addition, the additives carried by BMPs may also be released in the process of migration, posing a threat to aquatic organisms

Secondly, biofilms can also be formed on the surface of BMPs. Napper and Thompson (2019) investigated the degradation of biodegradable plastic bags and conventional plastic bags (PE) in different natural environments. The results showed that biofilm appeared on the surface of all biodegradable plastic bags after submerged in the marine environment for 1 month. The findings suggest that microorganisms may be more likely to aggregate on the surface of BDPs. Microplastics as vectors of microorganisms have positive effects, including accelerating the degradation of plastics and the transfer of bioenergy, and promoting ecological linkages. However, some studies have found that pathogenic bacteria are found on the surface of microplastics and occupy the main ecological niche on some particles, which may affect the safety of water quality and cause a potential threat to human health (Zettler et al. 2013). The biofilm attached to the microplastics is extremely complex and contains many genes, which may lead to gene exchange between the biofilm communities or between the biofilm communities and the surrounding communities. Due to the strong variability of bacterial genes, gene exchange between communities can be achieved by horizontal 
Fig. 3 The illustration of the multiple vector function of BMPs in the environment (BPA, Bisphenol A; PAHs, Polycyclic aromatic hydrocarbons)

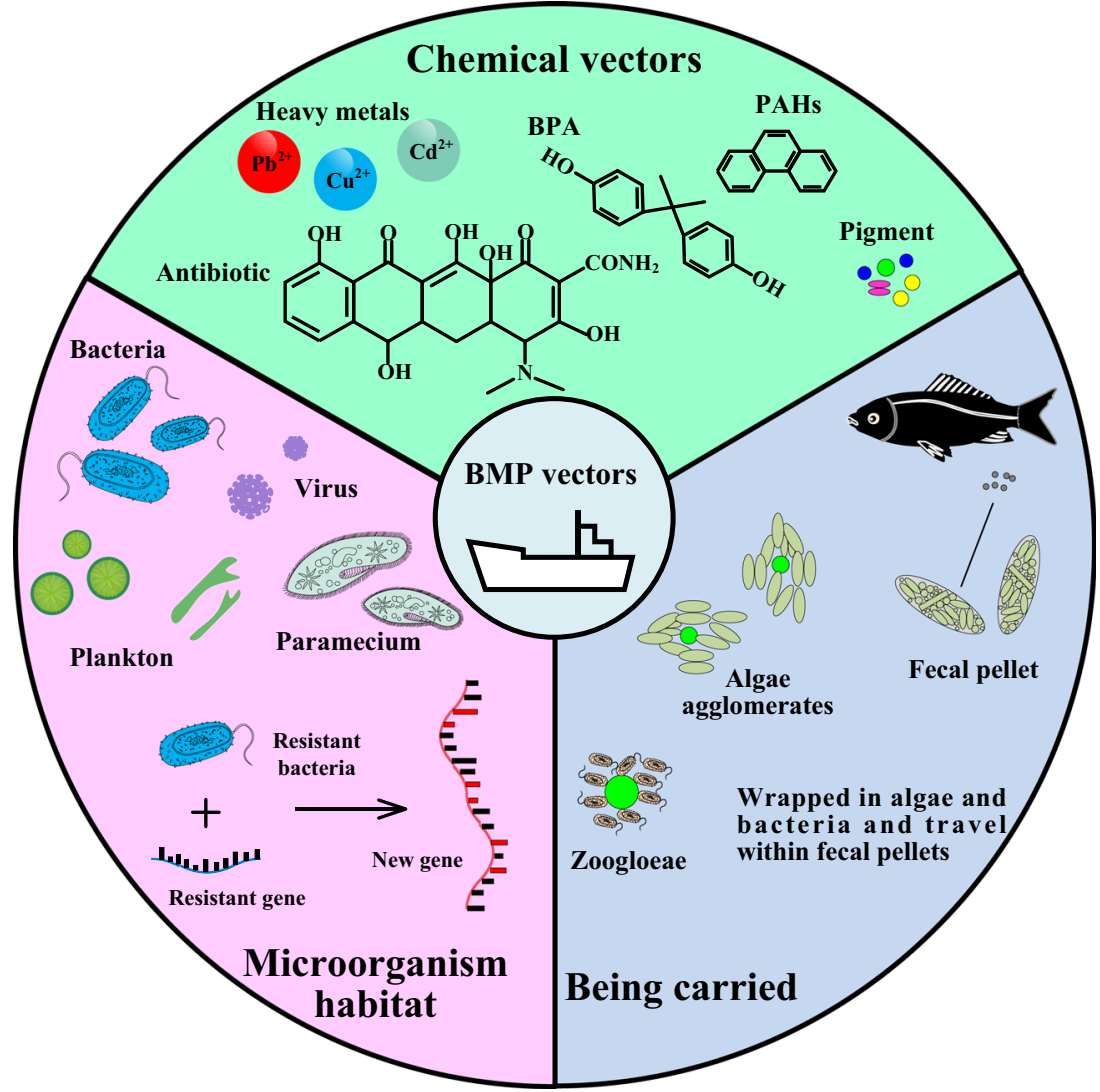

gene transfer, such as the absorption and utilization of DNA in the environment, gene transformation between cells, and gene transduction through phage (Fig. 3). Therefore, microplastic particles are not only the carrier of microbial diffusion and migration but also the carrier of gene exchange and transformation. Moreover, microplastic particles rich in the biofilm are efficient food sources for low trophic organisms such as zooplankton larvae and fish larvae. In the oligotrophic sea area, herbivorous marine animals may prefer to eat microplastic particles to obtain more food in order to survive, and microplastics have adverse effects on their growth and survival (Shen et al. 2019b). Compared with the conventional microplastics, biofilms can also be formed on the surface of BMPs, and there are many kinds of microorganisms in the biofilm, which may also become the primary prey of aquatic organisms.

Thirdly, BMPs can also be transported by organisms in the environment. In most cases, high-density microplastics sink, while low-density microplastics float and stay on the surface of the water. However, with the colonization of microorganisms, the hydrophobicity of microplastics decreases, the particle density is modified, and the buoyancy decreases gradually, which affects its vertical distribution in water. For example, many algae excrete extracellular polysaccharides, which can form aggregates of sticky particles under the action of turbulence, and microplastics can be encapsulated into these algal aggregates (Fig. 3). These agglomerated algal bodies are important vehicles for the vertical transportation of marine debris to the bottom of the ocean. Therefore, microplastics may also be transported vertically to the bottom of the water body in this way, resulting in different ecological effects. In addition, when microplastics are ingested by zooplankton and fish, the density and structural integrity of excreted feces will be changed, which will affect the vertical carbon flux of the aquatic ecosystem (Fig. 3).

However, at present, the research on BMPs as vectors for pollutants and microorganisms has just begun, and many conclusions are based on the inference stage. Our understanding of the interaction between BMPs and microbial communities on them is limited, and further research is needed. The identification of the main microbial communities, the population composition of different water environments, and the impact of microorganisms on the BMPs will become the key issues in the future.

\section{Would BMPs pose greater potential risks than microplastics to the environment and human health?}

Developing BP may be one important measure to control global white pollution (waste plastic pollution). But, BDPs 
may inevitably have the potential impact on the environment in the process of use. BDPs also can be decomposed into microplastics and nanoplastics, and researchers have begun to investigate the potential effects of BMPs on the environment (Green et al. 2021; Taylor et al. 1994). Green et al. (2016) studied the effects of polylactic acid microplastic (PLA) on benthic community growth richness and diversity, and the findings showed that the high concentration stress in sediments and the increase of respiration rate caused by PLA have adverse effects on biodiversity. Straub et al. (2017) compared the adsorption behavior of polyhydroxybutyrate microplastic (PHB) and polymethylmethacrylate microplastic (PMMA) in Gammarus fossarum. The findings suggested that PHB also had an ecotoxicological response as PMMA. The assimilation efficiency of Gammarus fossarum was obviously affected by the treatment of different particle sizes (32250 $\mu \mathrm{m})$, and the increase of wet weight was significantly reduced. The conclusions showed that BMPs would have appreciably less physiological effects on freshwater amphipods than MPs. In addition, Shruti and Kutralam-Muniasamy (2019) described in depth the potential impact of BMP on the ecosystem and aquatic organisms. A research carried out by GonzálezPleiter et al. (2019) studied the potential ecotoxicity impacts of secondary PHB nanoplastics on three representative aquatic organisms (Chlamydomonas reinhardtii, Anabaena sp., and Daphnia magna). Secondary PHB nanoplastics (25-100 mg $\mathrm{L}^{-1}, 200 \mathrm{~nm}$ ) used in the research were made of PHB microplastics. The findings demonstrated that the physical parameters related to the cell growth of three aquatic organisms were significantly decreased by the treatment of PHB nanoplastics. Moreover, Magni et al. (2020) studied the sublethal effects of polyvinyl chloride (PVC) microplastic and BMP (Mater-Biß HF03V2) on a freshwater biological model Dreissena polymorpha. The results showed that both PVC and Mater-Biß HF03V2 do not have adverse effects on exposed organisms, which may be due to its very effective protection mechanism. The authors also reported that both PVC and Mater-Bi® HF03V2 were found in the pseudo-feces, implying that Dreissena polymorpha has a very good mechanism to remove microplastics. Therefore, these studies demonstrated that biological contact with conventional and biodegradable microplastics and nanoplastics also showed similar ecotoxicity effects to experimental organisms and also indicate that BDPs are not completely safe for the environment. Evidence showed that there are bacteria, viruses, phytoplankton, and other organisms on the BMPs. Under the same conditions, according to the principle of predation, the predator will preferentially eat the particles with more food sources so as to improve the efficiency of predation. Especially in the low concentration of chlorophyll-a, the competition of zooplankton is fierce. The BMPs which spread from the eutrophic sea area to the poor nutrient sea area may carry rare food resources. However, the existing research on the ecotoxicity effect of BMPs has just started (Anderson and Shenkar 2021). Although it has been reported that BMPs can be preyed by microorganisms, there is still a lack of in-depth research on the cumulative effect of BMPs in food chain/web. Whether it has the effect of biomagnification, and whether it will enter the human body along the food chain/web and produce potential ecological hazards are also still lack of in-depth research.

\section{The road ahead}

With the continuous improvement of BP production, the widespread distribution and accumulation of BMPs in aquatic ecosystems have attracted public attention. From the perspective of research status, it is urgent to carry out the following aspects in the future:

(1) To study whether BMPs persist in the environment for a long time

First, measures should be taken to formulate biodegradability standards and specifications of BDPs. The degradation standard should not only be at the macro level but also consider whether microplastics and nanoplastics are produced during the degradation. Biodegradability of plastics is always linked to a specific environment. For plastics to get a "biodegradable label," specific environments are tested (e.g., industrial composting conditions). These environments can be simulated and tested for via standard test methods, which are in place and setup by various standard test organizations like ISO, CEN, and ASTM International. There are specific pass/ fail criteria that a plastic needs to reach before it can get such a label. And even then, it only acquires the label for that specifically tested environment. Compared with large BDP debris, BMPs may be more harmful to aquatic organisms. Second, the evaluation of the degradation behavior of BMPs in the real environment is also needed. The conditions of soil, water, and atmosphere environment are different. The degradability of BMPs in three compartments has to be studied, and it is not enough to consider only one compartment. It is also necessary to consider aerobic and anaerobic conditions, biodegradability, and physical degradability. With the increasing public awareness of environmental protection, more and more BDPs will be used in agriculture and daily life. These products will undoubtedly enter the natural environment. Therefore, to study whether BMPs persist in the environment for a long time is necessary.

(2) To explore the carrier function of BMPs and to evaluate the ecological effects of BMPs

The research of marine BMPs as biological carriers is a new research topic. The carrier effect of BMPs in the aquatic 
ecosystem may exceed its own toxicological effect. The mechanism of how BMPs can change the enrichment and toxicity of coexisting pollutants is not clear, which needs further research and exploration. It is necessary to accelerate the research process to understand the role of BMPs in microbial migration and the carbon cycle. Additionally, the aggregation of BMPs as vectors may induce gene exchange among microorganisms, resulting in the uncontrollable transmission of antibiotic-resistant genes and the large-scale outbreak of drug-resistant pathogens. The migration and diffusion of BMPs as carriers may promote the spread of harmful algal species, pathogenic bacteria, and drug-resistant bacteria and rapidly propagate when they reach certain suitable conditions. The combination of BMPs and biofilm may enhance the predation of organisms, which may promote the energy flow, material flow, and information flow rate of the ecosystem, but may also aggravate the toxicological and pathological reactions of marine organisms feeding on BMPs. Its severity may be far greater than the consequences of conventional microplastics as carriers. However, it is still unknown how microorganisms are adsorbed on vectors, what are the advantages of community structure and function, the impact of community aggregation on predators, the probability of gene exchange, and the diffusion mechanism of vectors as pests. In addition, the selection of organisms and their competitive adsorption is not known when both conventional and biodegradable microplastics are present. Field and laboratory simulation experiments are needed to further explore the selectivity of pollutants and organisms to conventional and biodegradable microplastics, and to further explore the aggregation and diffusion mechanism of these vectors.

\section{(3) To establish the toxicity test method of BMPs and further test to determine the toxicity effect of BDPs}

Due to the different physical and chemical properties of BMPs and common pollutants, it is necessary to establish a standard toxicity test method (such as OECD test methods). There are many studies on the ingestion of BMPs by aquatic organisms, but few studies on their toxic effects, mainly concentrated on a few kinds of aquatic organisms, and the toxicity endpoint indicators are not comprehensive enough. In the future, it is necessary to carry out more systematic ecotoxicological research on the basis of further understanding the toxic mechanism of BMPs to these organisms. The exposure concentration used in the study is usually higher than the environmental concentration, and the exposure time is short. Acute toxicity tests often do not provide comprehensive information, so the research on low-dose long-term exposure of BMPs needs to be strengthened. The effects of exposure test conditions (acid-base, temperature, and organic matter content) on the morphology and migration of BMPs, and the interaction between BMPs and biomacromolecules after entering the organism have not been clearly defined, which should be paid attention to in future research.

Interest and contribution The authors declare no competing interests. Can Wang and Jiefa Yu contributed equally in this work.

Can Wang and Jiefa Yu: conceptualization, methodology, investigation, writing — original draft.

Ying Lu: methodology, investigation, review \& editing.

Di Hua: methodology, investigation, review \& editing. Xiao Wang: methodology, investigation, review \& editing. Xuehua Zou: conceptualization, methodology, writing - review \& editing, resources.

Data availability The datasets used or analyzed during the current study are available from the corresponding author on reasonable request

\section{Declaration}

Ethical approval This manuscript was only submitted on Environmental Science and Pollution Research.

The authors make sure they have permissions for the use of software, questionnaires/(web) surveys and scales in their studies (if appropriate). This research may not be misapplied to pose a threat to public health or national security. There was no animal experiment in this manuscript.

Consent to participate and publish Results in this manuscript were presented clearly, honestly, and without fabrication, falsification or inappropriate data manipulation (including image based manipulation).

All co-authors have seen and approved the manuscript and have agreed to its submissions for publication

\section{References}

Anderson G, Shenkar N (2021) Potential effects of biodegradable singleuse items in the sea: polylactic acid (PLA) and solitary ascidians. Environ Pollut 268:115364

Catarino AI, Macchia V, Sanderson WG, Thompson RC, Henry TB (2018) Low levels of microplastics (MP) in wild mussels indicate that MP ingestion by humans is minimal compared to exposure via household fibres fallout during a meal. Environ Pollut 237:675-684

Cole M, Lindeque P, Fileman E, Halsband C, Goodhead R, Moger J, Galloway TS (2013) Microplastic ingestion by zooplankton. Environ Sci Technol 47:6646-6655

Gasperi J, Wright SL, Dris R, Collard F, Mandin C, Guerrouache M, Langlois V, Kelly FJ, Tassin B (2018) Microplastics in air: are we breathing it in? Current Opinion in Environmental Science \& Health $1: 1-5$

González-Pleiter M, Tamayo-Belda M, Pulido-Reyes G, Amariei G, Leganés F, Rosal R, Fernández-Piñas F (2019) Secondary nanoplastics released from a biodegradable microplastic severely impact freshwater environments. Environ Sci Nano 6:1382-1392

González-Pleiter M, Edo C, Aguilera Á, Viúdez-Moreiras D, PulidoReyes G, González-Toril E, Osuna S, de Diego-Castilla G, Leganés F, Fernández-Piñas F, Rosal R (2021) Occurrence and transport of microplastics sampled within and above the planetary boundary layer. Sci Total Environ 761:143213 
Gouin T, Roche N, Lohmann R, Hodges G (2011) A thermodynamic approach for assessing the environmental exposure of chemicals absorbed to microplastic. Environ Sci Technol 45:1466-1472

Green DS, Boots B, Sigwart J, Jiang S, Rocha C (2016) Effects of conventional and biodegradable microplastics on a marine ecosystem engineer ( Arenicola marina ) and sediment nutrient cycling. Environ Pollut 208:426-434

Green DS, Jefferson M, Boots B, Stone L (2021) All that glitters is litter? Ecological impacts of conventional versus biodegradable glitter in a freshwater habitat. J Hazard Mater 402:124070

Gregory MR (2009) Environmental implications of plastic debris in marine settings-entanglement, ingestion, smothering, hangers-on, hitch-hiking and alien invasions. Philosophical Transactions of the Royal Society of London 364:2013-2025

Haider TP, Völker C, Kramm J, Landfester K, Wurm FR (2019) Plastics of the future? The impact of biodegradable polymers on the environment and on society. Angew Chem Int Edit 58:50-62

Hamilton LA et al. (2019) Plastic \& climate: the hidden costs of a plastic planet center for international environmental law (CIEL). https:// www.ciel.org/plasticandclimate

Hu D, Shen M, Zhang Y, Li H, Zeng G (2019) Microplastics and nanoplastics: would they affect global biodiversity change? Environ Sci Pollut Res 26:19997-20002

Koelmans AA, Bakir A, Burton GA, Janssen CR (2016) Microplastic as a vector for chemicals in the aquatic environment: critical review and model-supported re-interpretation of empirical studies. Environ Sci Technol 50:3315-3326

Law KL, Thompson RC (2014) Microplastics in the seas. Science 345: 144-145

Lobelle D, Cunliffe M (2011) Early microbial biofilm formation on marine plastic debris. Mar Pollut Bull 62:197-200

Lott C, Eich A, Unger B, Makarow D, Battagliarin G, Schlegel K, Lasut MT, Weber M (2020) Field and mesocosm methods to test biodegradable plastic film under marine conditions. Plos One 15: e0236579

Ma Y, Huang A, Cao S, Sun F, Wang L, Guo H, Ji R (2016) Effects of nanoplastics and microplastics on toxicity, bioaccumulation, and environmental fate of phenanthrene in fresh water. Environ Pollut 219:166-173

Machado AAD, Kloas W, Zarfl C, Hempel S, Rillig MC (2018) Microplastics as an emerging threat to terrestrial ecosystems. Global Change Biol 24:1405-1416

Magni S, Bonasoro F, Torre CD, Parenti CIC, Maggioni D, Binelli A (2020) Plastics and biodegradable plastics: ecotoxicity comparison between polyvinylchloride and Mater-Bi ${ }^{\circledR}$ micro-debris in a freshwater biological model. Sci Total Environ 720:137602

Napper IE, Thompson RC (2019) Environmental deterioration of biodegradable, oxo-biodegradable, compostable, and conventional plastic carrier bags in the sea, soil, and open-air over a 3-year period. Environ Sci Technol 53:4775-4783

Narancic T, O'Connor KE (2019) Plastic waste as a global challenge: are biodegradable plastics the answer to the plastic waste problem? Microbiol-Sgm 165:129-137

Nazareth M, Marques MR, Leite MC, Castro ÍB (2019) Commercial plastics claiming biodegradable status: is this also accurate for marine environments? J Hazard Mater 366:714-722

Oßmann BE, Sarau G, Holtmannspötter H, Pischetsrieder M, Christiansen SH, Dicke W (2018) Small-sized microplastics and pigmented particles in bottled mineral water. Water Res 141:307-316

Pico Y, Barcelo D (2019) Analysis and prevention of microplastics pollution in water: current perspectives and future directions. ACS Omega 4:6709-6719

Rochman CM, Kurobe T, Flores I, Teh SJ (2014) Early warning signs of endocrine disruption in adult fish from the ingestion of polyethylene with and without sorbed chemical pollutants from the marine environment. Sci Total Environ 493:656-661

Shen M, Zhang Y, Zhu Y, Song B, Zeng G, Hu D, Wen X, Ren X (2019a) Recent advances in toxicological research of nanoplastics in the environment: a review. Environ Pollut 252:511-521

Shen M, Zhu Y, Zhang Y, Zeng G, Wen X, Yi H, Ye S, Ren X, Song B (2019b) Micro(nano)plastics: unignorable vectors for organisms. Marine Pollution Bulletin 139:328-331

Shen M, Huang W, Chen M, Song B, Zeng G, Zhang Y (2020) (Micro)plastic crisis: un-ignorable contribution to global greenhouse gas emissions and climate change. J Clean Prod 254:120138. https:// doi.org/10.1016/j.jclepro.2020.120138

Shen M, Song B, Zeng G, Zhang Y, Teng F, Zhou C (2021) Surfactant changes lead adsorption behaviors and mechanisms on microplastics. Chem Eng J 405:126989

Shruti V, Kutralam-Muniasamy G (2019) Bioplastics: missing link in the era of microplastics. Sci Total Environ 697:134139

Straub S, Hirsch PE, Burkhardt-Holm P (2017) Biodegradable and petroleum-based microplastics do not differ in their ingestion and excretion but in their biological effects in a freshwater invertebrate gammarus fossarum. Int J Environ Res Pu He 14:774

Taylor M, Daniels A, Andriano K, Heller J (1994) Six bioabsorbable polymers: in vitro acute toxicity of accumulated degradation products. J Appl Biomater 5:151-157

Thompson RC, Olsen Y, Mitchell RP, Davis A, Rowland SJ, John AW, McGonigle D, Russell AE (2004) Lost at sea: Where is all the plastic? Science 304:838-838

Tong H, Hu X, Zhong X, Jiang Q (2021) Adsorption and desorption of triclosan on biodegradable polyhydroxybutyrate microplastics. Environ Toxicol Chem 40:72-78

Wang W, Ndungu AW, Li Z, Wang J (2016) Microplastics pollution in inland freshwaters of China: a case study in urban surface waters of Wuhan, China. Sci Total Environ 575:1369-1374

Wang J, Wang MX, Ru SG, Liu XS (2019) High levels of microplastic pollution in the sediments and benthic organisms of the South Yellow Sea, China. Sci Total Environ 651:1661-1669

Wei X-F, Bohlén M, Lindblad C, Hedenqvist M, Hakonen A (2021) Microplastics generated from a biodegradable plastic in freshwater and seawater. Water Res 198:117123

Xia W, Rao Q, Deng X, Chen J, Xie P (2020) Rainfall is a signi ficant environmental factor of microplastic pollution in inland waters. Sci Total Environ 732:139065

Zarfl C, Matthies M (2010) Are marine plastic particles transport vectors for organic pollutants to the Arctic. Mar Pollut Bull 60:1810-1814

Zettler ER, Mincer TJ, Amaral-Zettler LA (2013) Life in the "plastisphere": microbial communities on plastic marine debris. Environ SciTechnol 47:7137-7146

Zhang W, Dong Z, Zhu L, Hou Y, Qiu Y (2020) Direct observation of the release of nanoplastics from commercially recycled plastics with correlative raman imaging and scanning electron microscopy. Acs Nano 14:7920-7926

Zhongyan Puhua Company (2014) Report of indepth research and investment strategy planning on china mbiodegradable plastics industry 2015-2020. https://www.chinairn.com/report/20141112/ 155443213.html

Zuo L-Z, Li HX, Lin L, Sun YX, Diao ZH, Liu S, Zhang ZY, Xu XR (2019) Sorption and desorption of phenanthrene on biodegradable poly (butylene adipate co-terephtalate) microplastics. Chemosphere 215:25-32

Publisher's note Springer Nature remains neutral with regard to jurisdictional claims in published maps and institutional affiliations. 